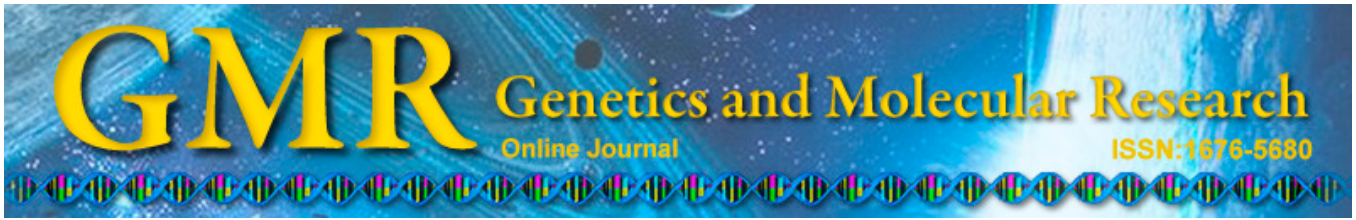

\title{
Opg/Rankl mRNA dynamic expression in the bone tissue of ovariectomized rats with osteoporosis
}

\author{
C.W. Li ${ }^{1 *}$, B. Liang ${ }^{2 *}$, X.L. $\mathrm{Shi}^{3}$ and H. Wang ${ }^{3 *}$ \\ ${ }^{1}$ Zhejiang Chinese Medical University, Hangzhou, China \\ ${ }^{2}$ Haiyan Hospital of Traditional Chinese Medicine of Zhejiang Province, \\ Jiaxing, China \\ ${ }^{3}$ The Second Affiliated Hospital of Zhejiang Chinese Medical University, \\ Hangzhou, China
}

*These authors contributed equally to this study.

Corresponding author: X.L. Shi

E-mail: xldoccn@163.com

Genet. Mol. Res. 14 (3): 9215-9224 (2015)

Received January 29, 2015

Accepted May 22, 2015

Published August 10, 2015

DOI http://dx.doi.org/10.4238/2015.August.10.1

\begin{abstract}
We established animal models of osteoporosis in ovariectomized rats to detect osteoprogerin $(\mathrm{Opg}) /$ receptor activator of nuclear factor- $\mathrm{kB}$ ligand (Rankl) mRNA expression levels in the tibias and serum estradiol concentrations at different time points. Sixty Sprague-Dawley female rats were randomly selected and divided into an ovariectomized (OVX) group and sham-operated (SHAM) group. In the SHAM group, only a small amount of abdominal fat and tissues was removed from the rats. Ten rats in each group were sacrificed at 0 , 6 , and 12 months after establishing the animal models (12 weeks). Opg mRNA expression and serum estradiol concentration in the OVX group were significantly lower than those in the SHAM group $(\mathrm{P}<0.05)$. In contrast, Rankl mRNA expression in the OVX group was significantly higher than that in the SHAM group $(\mathrm{P}<0.05)$. In the OVX group, Opg mRNA expression and serum estradiol concentrations decreased
\end{abstract}


significantly from 0 to 12 months $(\mathrm{P}<0.05)$, whereas Rankl mRNA expression increased significantly $(\mathrm{P}<0.05)$. Opg mRNA expression and serum estradiol concentrations in the OVX group continually decreased, whereas Rankl mRNA expression continually increased. The $\mathrm{Opg} / \mathrm{Rankl}$ ratio showed a decrease. The OPG/RANKL ratio may be a key factor affecting the osteoblast-mediated reaction.

Key words: Osteoprotegerin; Ovariectomized rats; Receptor activator of nuclear factor- $\kappa \mathrm{B}$ ligand

\section{INTRODUCTION}

The incidence of osteoporosis (OP) in China, the most populous country in the world, increases annually. Epidemiological investigation has revealed that the incidence rates of OP among adults aged 50-60, 60-70, and 70-80 years are 21,58 , and $100 \%$, respectively (Wada et al., 2006). OP is one of the most common human diseases worldwide. Type I or post-menopausal OP (PMOP) belongs to the high conversion rate type of OP, whereas type II or senile OP belongs to the low conversion rate type of OP. In some cases such as weight loss, brake, and prolonged stay in bed, the long-term use of hormones can cause the high conversion rate type of OP, leading to loss of cancellous bone mass. The rate of loss increases with age in post-menopausal women. Patients with type II OP, cancellous bone, and cortical bone show different loss levels, with the rate of loss increasing by $50-70 \%$ in post-menopausal women with OP aged $>65$ years (Hofbauer et al., 2009). The prevention and treatment of OP have received considerable attention, with the aim of reducing the incidence of osteoporotic fractures and improving patients' quality of life. Targeting gene research is particularly important for treating OP.

The receptor activator of nuclear factor- $\kappa \mathrm{B}$ ligand (RANKL)/receptor activator of nuclear factor- $\mathrm{\kappa B}$ (RANK)/osteoprotegerin (OPG) system is an important signaling pathway in osteoclast differentiation. Bone formation and resorption mediated by osteoblasts and osteoclasts maintain bone metabolic balance and integrity. Numerous hormones and cytokines are involved in regulating osteoblasts and osteoclasts, among which the RANKL/RANK/OPG system plays a key role. Osteoblasts and bone marrow stromal cells secrete RANKL, which combines with RANK on osteoclasts/broken bone precursor cells to mediate osteoclast maturation and increase bone resorption (Tanaka et al., 2011). Simultaneously, they express OPG, which competitively combines with RANKL to inhibit osteoclast generation and maturation ( Marie and Halbout, 2008). OPG also promotes osteoclast apoptosis to inhibit bone resorption (Reid and Holen, 2009). Moreover, the OPG/RANKL/RANK system regulates osteoclast differentiation, activation, and apoptosis to promote bone formation by advancing contact with osteogenic cells (Berenson et al., 2006). Among the 3 proteins, the OPG/RANKL ratio may be a key factor affecting the osteoblast-mediated reaction. A greater OPG/RANKL ratio has been correlated with stronger osteoblast-mediated function (Macmillan et al., 2001). The OPG/ RANKL ratio in osteoporotic bone tissue is much lower than that in normal human bone tissue, suggesting that osteoclast formation is greater in osteoporotic bone tissue than in normal bone tissue. Osteoclast formation is likely the main pathological mechanism of OP. Studies have focused on the relationship between PMOP and the RANKL/RANK/OPG system during the menopausal period. However, little is known regarding the relationship between PMOP and the RANKL/RANK/OPG system during the post-menopausal period. In the present study, 
experimental and control rat groups were observed at different periods of 0,6 , and 12 months after 12 weeks of serum E2 level determination and bone tissue $\mathrm{Opg} / \mathrm{Rankl}$ dynamic variation study. The results of the present study may serve as a theoretical foundation for targeted gene therapy to treat PMOP.

\section{MATERIAL AND METHODS}

\section{Subjects}

Sixty 3-month-old female Sprague-Dawley (SD) rats were randomly selected. This study was carried out in strict accordance with the recommendations in the Guide for the Care and Use of Laboratory Animals of the National Institutes of Health. The animal use protocol was reviewed and approved by the Institutional Animal Care and Use Committee of the Second Affiliated Hospital of Zhejiang Chinese Medical University.

\section{Methods}

Female SD rats were randomly divided into an ovariectomized (OVX) group $(\mathrm{N}=30)$ and a sham-operated (SHAM) group $(\mathrm{N}=30)$. In the SHAM group, only a small amount of abdominal fat and tissues was removed. Ten rats from each group were sacrificed at 0,6 , and 12 months after establishing the OP models (12 weeks). Opg/Rankl mRNA expression levels in the tibias and serum estradiol (E2) concentrations were then detected.

The primer sequences of glyceraldehyde 3-phosphate dehydrogenase (GAPDH) were 5'-CAAGGTCATCCATGACAACTTTG-3' (forward) and 5'-GTCCACCACCCTGTTGCTG TAG-3' (reverse); those of rat $O P G$ were 5'-TGTGGAATAGATGTCACCCTGTGC-3' (forward) and 5'-CACAGAGGTCAATGTCTTGGATGATC-3' (reverse); and those of rat RANKL were 5'-GCTTCTCAGGAGTTCCAGCTATGAT-3' (forward) and 5'-CGTTGCTTAACGTC ATGTTAGAGATCT (reverse).

\section{Statistical analysis}

The SPSS version17.0 software package was used for statistical analysis (SPSS, Inc.; Chicago, IL, USA), and differences with $\mathrm{P}<0.05$ were considered to be statistically significant.

\section{RESULTS}

The system and conditions of real-time polymerase chain reaction (RT-PCR) complied with the requirements of RT-PCR, as shown in Figure 1. The melting curves of the RTPCR product revealed a single dissolved peak (Figure 2). This demonstrated that the RT-PCR for the gene was specific.

\section{Opg/Rankl mRNA expression level of the tibias and serum E2 concentration}

Opg mRNA expression levels of the tibias and serum E2 concentrations in the OVX group were significantly lower than those in the SHAM group $(\mathrm{P}<0.05)$ (Figures 3 and 4). In contrast, Rankl mRNA expression levels in the OVX group were significantly higher than 
those in the SHAM group $(\mathrm{P}<0.05)$ (Figure 5). In the OVX group, the Opg mRNA expression levels and serum E2 concentrations decreased significantly from 0 to 12 months $(\mathrm{P}<0.05)$. However, the Rankl mRNA expression levels increased significantly $(\mathrm{P}<0.05)$.
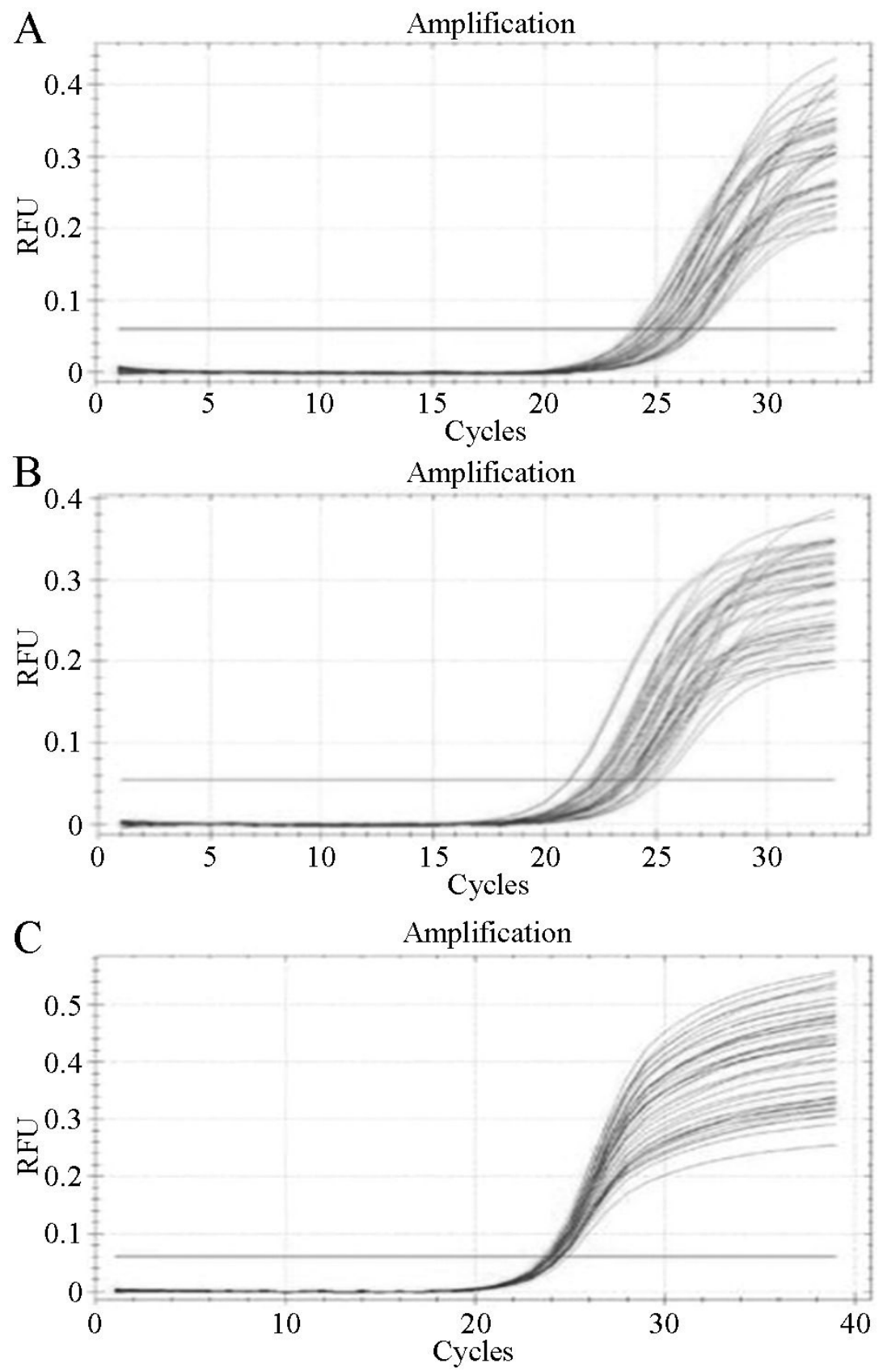

Figure 1. RT-PCR amplification curves of $O p g(\mathbf{A}), \operatorname{Rankl}(\mathbf{B})$, and $G A P D H(\mathbf{C})$. 

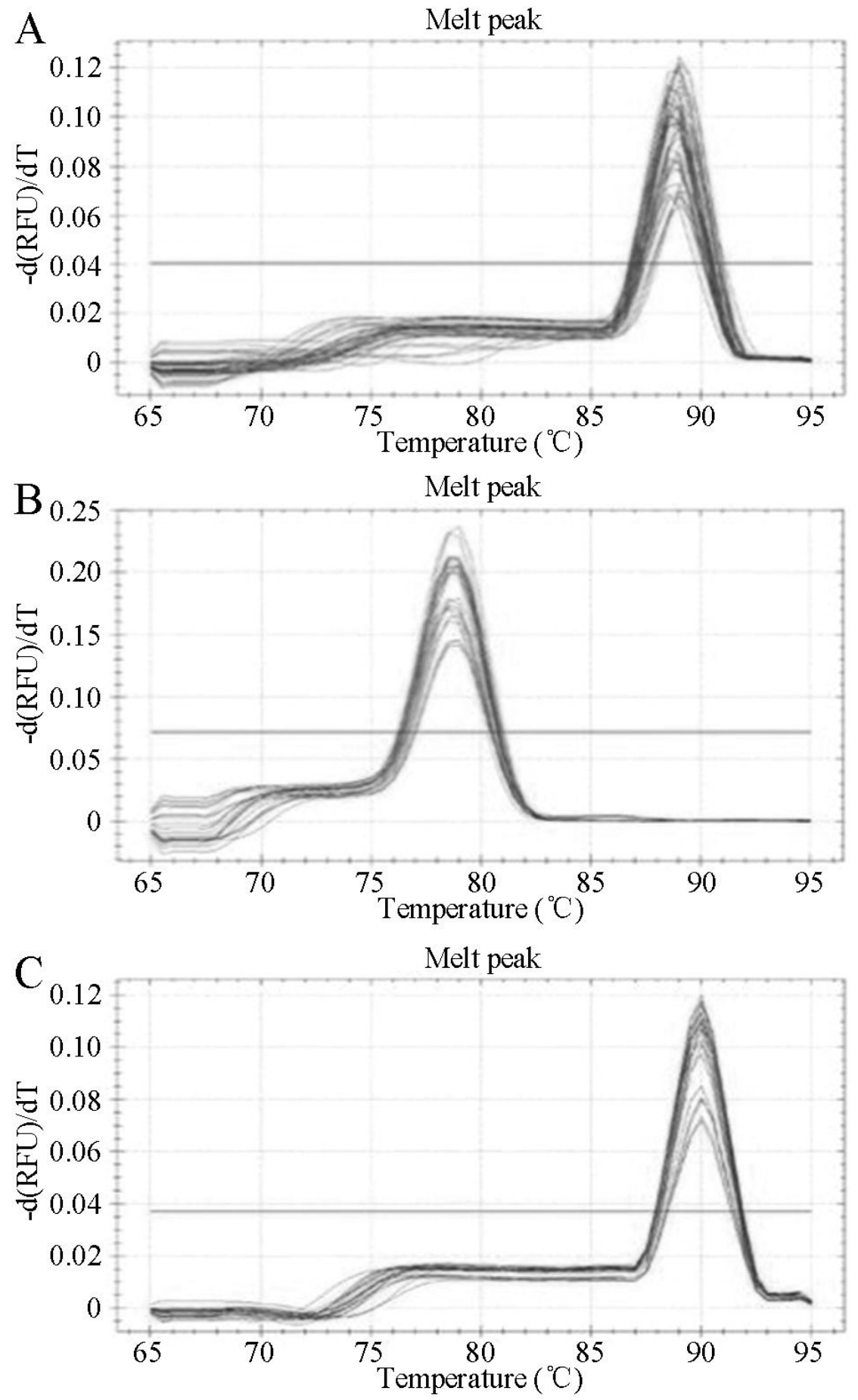

Figure 2. RT-PCR melting curves of $O p g(\mathbf{A}), \operatorname{Rankl}(\mathbf{B})$, and $G A P D H(\mathbf{C})$. 


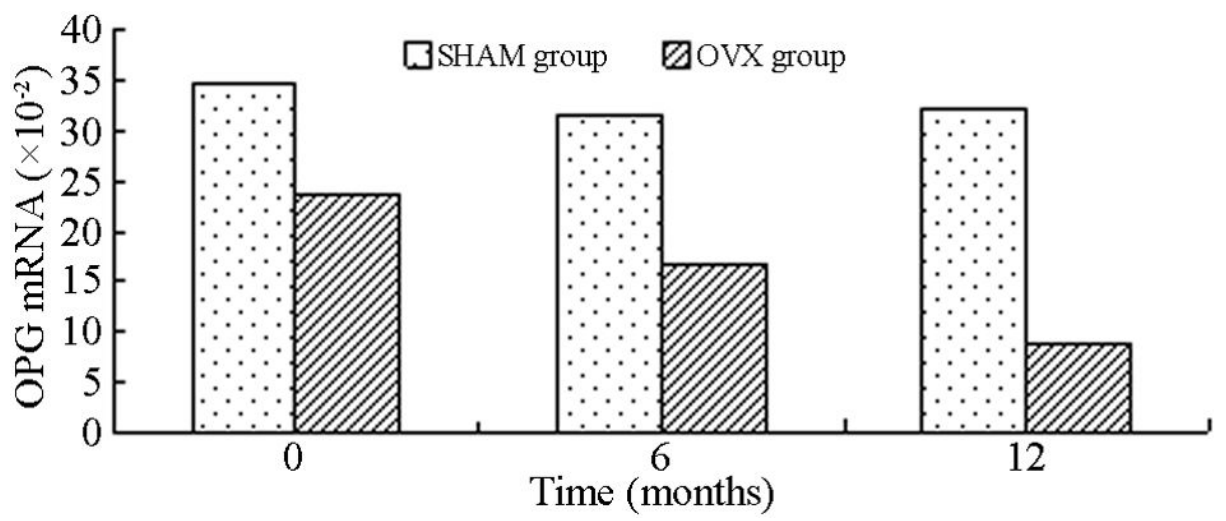

Figure 3. Changes in Opg mRNA expression in the tibia.

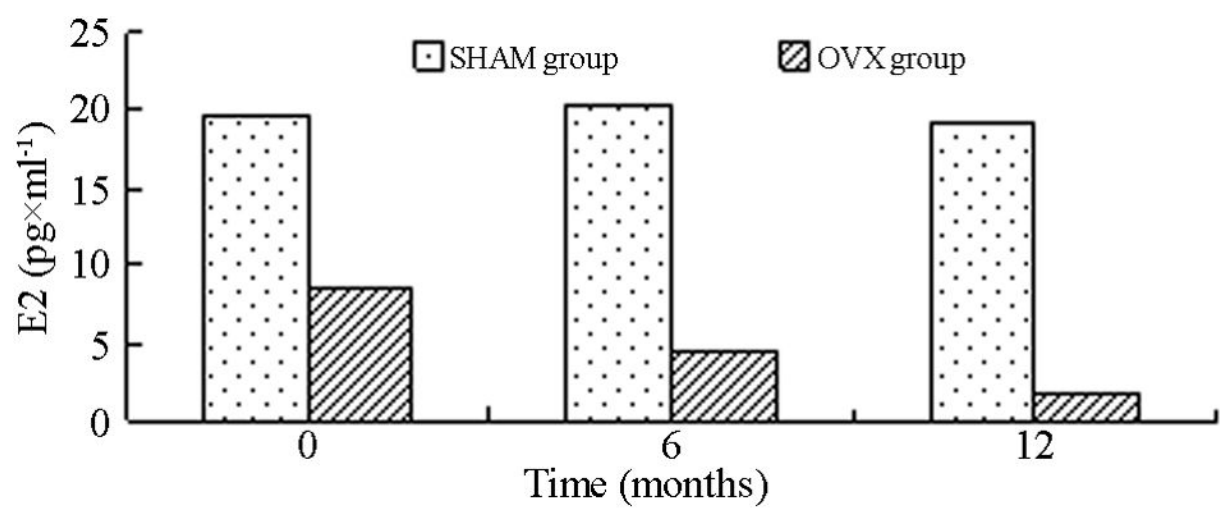

Figure 4. Changes in serum E2 concentrations.

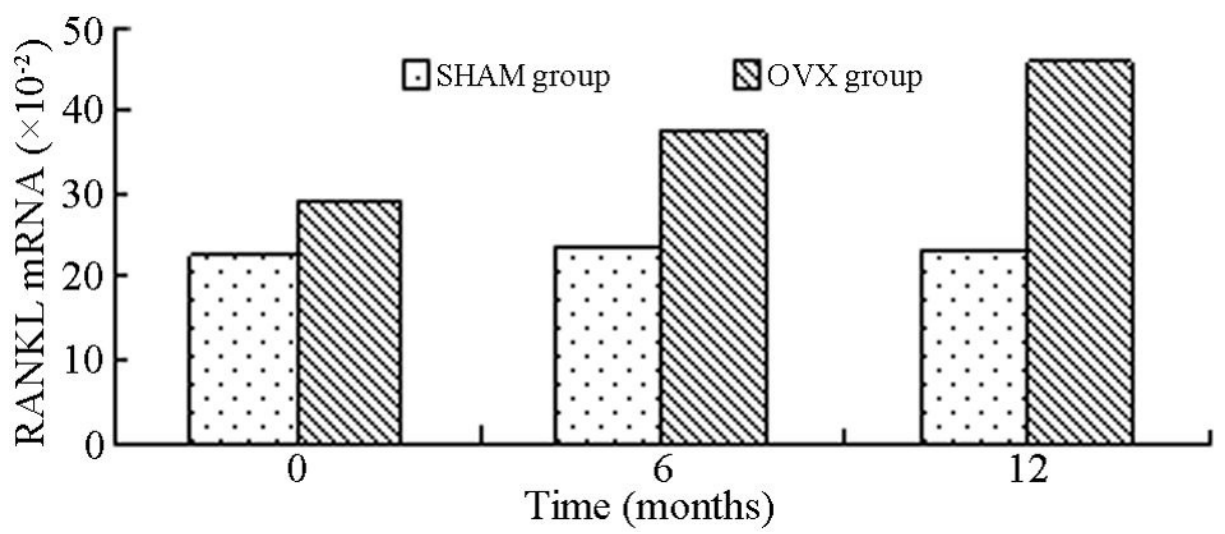

Figure 5. Changes in Rankl mRNA expression in the tibia. 


\section{DISCUSSION}

Rat OP models were subjected to ovariectomy, retinoic acid lavage, hormonal induction, restricted diet, and restricted physical movement. Bilateral ovariectomy causes estrogen loss and induces OP. This method can be employed as a reliable animal model of post-menopausal OP (Pobeha et al., 2010). In 1969, Womski successfully established an animal model of postmenopausal OP through bilateral ovariectomy in rats (Kalu, 1991). Since then, this rat model has been widely used because of its stability, reliability, high success rate, and good repeatability. Within 100 days after ovariectomy, bone loss rapidly occurs. Bekker et al. (2005) reported significant differences in the lumbar spine and femoral bone mineral density 8-9 weeks after ovariectomy, suggesting that the model was successful. The United States Food and Drug Administration and World Health Organization recommended that ovariectomized rats are the best animal models for studies examining PMOP (Bonnelye et al., 2002). Moreover, the Food and Drug Administration also suggested that the modeling time for female ovariectomized rats should be 7-12 weeks in PMOP studies. In the present study, ovariectomized female SD rats were selected, using a modeling time of 12 weeks, to ensure the successful modeling of PMOP. Simultaneously, vaginal smears were continually collected for 3 days. No keratin was found in the ovariectomized rat group, confirming that bilateral ovariectomy was successful.

In 1997, OPG was discovered by 2 laboratories in the United States and Japan. OPG (molecular weight: 60 or $119 \mathrm{kD}$ ), a member of the tumor necrosis factor (TNF) receptor superfamily, is a secretory glycoprotein active in its monomer and dimer forms. The precursor of OPG is a polypeptide composed of 401 amino acids. Cleavage of 1 of the 21 amino acids results in formation of a mature soluble protein composed of 380 amino acids. Aside from its high expression in osteoblasts, OPG is widely expressed in the heart, kidney, liver, spleen, and bone marrow (Shimizu-Ishiura et al., 2002). As a decoy receptor, OPG can competitively bind to RANKL and TNF-related apoptosis-inducing ligand to block the interaction between RANKL with RANK. Therefore, OPG inhibits the differentiation and maturation of osteoclasts and induces osteoclast apoptosis (Sinclair and Elliott, 2005).

RANK, a member of the TNF receptor superfamily $11 \mathrm{~A}$, is a type I transmembrane protein. The human RANK peptide is composed of 616 amino acids, including 28 amino acid signal peptides. At both the N- and C-terminal ends, RANK contains 21 amino acid residues in the extracellular region and 383 amino acid restudies in the intracellular region (Wang et al., 2008). RANK is mainly expressed in monocyte and macrophage lineage cells (such as osteoclast precursor cells, lymphocytes, dendritic cells, and osteoblasts), as well as in the skeletal muscle, trabecular bone, thymus, liver, rectum, and small intestine. RANK is the only target signal receptor associated with RANKL that promotes osteoclast differentiation. RANK combines with RANKL in osteoclasts and on the surface of progenitor cells, directly promoting the differentiation, activation, and maturation of osteoclasts and preventing osteoclast apoptosis.

The functions of the OPG/RANKL/RANK system are related to their expression in osteoblasts and osteoclasts. Osteoblasts and bone marrow stromal cells secrete RANKL, which combines with RANK on the osteoclast/broken bone precursor cells to mediate osteoclast maturation and increase bone resorption. Simultaneously, these cells express OPG, which competitively binds to RANKL to inhibit osteoclast generation and maturation. OPG also promotes osteoclast apoptosis to inhibit bone resorption. Moreover, OPG establishes contact with osteogenic cells to promote bone formation. Recent studies have found that the OPG/RANKL/RANK system regulates osteoclast differentiation, activation, and apoptosis (Berenson et al., 2006). 
The OPG/RANKL ratio may be a key factor affecting osteoblast-mediated reactions. A greater $\mathrm{OPG} / \mathrm{RANKL}$ ratio was correlated with stronger osteoblast-mediated functions.

The expression of bone tissue Opg mRNA in the OVX group was significantly lower than that in the SHAM group $(\mathrm{P}<0.05)$, with significant reductions observed at 6 and 12 months $(\mathrm{P}<0.01)$. In the OVX group, Opg mRNA expression levels significantly decreased from 0 to 12 months $(\mathrm{P}<0.05)$. At 12 weeks after ovariectomy, the expression of bone tissue Opg mRNA continuously decreased. This change is consistent with the serum concentration of E2, suggesting that expression of rat tibia bone Opg mRNA is closely related to changes in serum E2 concentration. Compared with the SHAM group, the Rankl mRNA expression level in the tibial bone in the OVX group increased significantly $(\mathrm{P}<0.01)$ and continuously. The $\mathrm{Opg} /$ Rankl ratio showed a narrowing trend. During the 12 months after ovariectomized rat post-menopause osteoporosis models were established, Opg mRNA expression in the tibia tissue and serum E2 continuously decreased, whereas Rankl mRNA increased. However, the end points of these changes remain unknown. These findings indicate that osteoporosis is progressively aggravated without intervention treatment (such as drugs) during this period.

The OPG/RANKL/RANK system is thought to be regulated by estrogen. This system is also thought to play a role PMOP. Estrogen affects the OPG/RANKL/RANK system through the following events. After estrogen binds to its receptors, osteoblastic OPG expression increases, the OPG/RANKL ratio increases, and osteoclast formation and function are inhibited (Thompson et al., 1995; Blair et al., 2007). In the OVX group, serum E2 concentrations decreased, Opg mRNA expression decreased, Rankl mRNA expression increased, and the $O p g / R a n k l$ ratio narrowed. These results are consistent with those of previous studies (Rogers et al., 2002; Saika et al., 2001). Estrogen reduces the expression of interleukin-1, interleukin 6 , TNF- $\alpha$, and prostaglandin E2, but increases the expression of transforming growth factor $\beta$ and calcitonin to control the OPG/RANKL ratio. Estrogen can decrease expression of the interleukin 6 and TNF- $\alpha$ genes in osteoblasts and bone marrow stromal cells. When the estrogen level is inadequate, interleukin 6 and TNF- $\alpha$ levels increase. Interleukin 6 mediated by gp130 increases the expression of $R A N K L$ mRNA. TNF- $\alpha$ mediated by prostaglandin E2 increases the expression of RANKL mRNA but decreases the expression of $O P G$ mRNA. RANKL can significantly strengthen the effect of TNF- $\alpha$. Through inactivation of the c-Jun N-terminal kinase pathway, estrogen suppresses RANKL-induced osteoclast differentiation (Hamdy, 2007). Decreased estrogen significantly increased the RANKL/OPG ratio, thereby increasing bone absorption. During the post-menopause phase, RANKL expression was increased, osteoclast differentiation began, and maturation signals were strengthened. However, RANKL weakened as OPG expression decreased, increasing bone absorption and leading to osteoporosis.

Determination of the role of the OPG/RANK/RANKL system in regulating bone metabolism created new avenues for the development of drugs to prevent and control OP. OPG or RANKL expression in the OPG/RANK/OPG signaling pathway has become the most relevant therapeutic target for osteolytic disease (Van Staa et al., 2000; Sasaki and Kusano, 2006; Tanaka, 2007). In a study by Syed and Khosla (2005), trabecular bone increased significantly in OPG-treated ovariectomized mice, but decreased in untreated ovariectomized mice. Compared with OPG, Fc-OPG showed higher stability and a longer half-life (Bord et al., 2003; Gao et al., 2009). Denosumab, the monoclonal antibody of RANKL, can inhibit RANKL by combining with its receptors. The specificity and half-life of denosumab are better than those of OPG. Denosumab also has no autoantibody. Hamdy (2006) and Caim et al. (2009) found 
that denosumab can prevent glucocorticoid-induced bone loss and can activate human $R A N K L$ gene knockout in mice. Therefore, the OPG/RANKL/RANK system provides an ideal target for preventing and treating bone diseases such as PMOP. However, whether these drugs have side effects should be further examined.

\section{Conflicts of interest}

The authors declare no conflict of interest.

\section{REFERENCES}

Bekker PJ, Holloway DL, Rasmussen AS, Murphy R, et al. (2005). A single-done placebo-controlled study of AMG 162, a full human monoclonal antibody to RANKL, in postmenopausal women. J. Bone Miner. Res. 20: 2275-2282.

Berenson JR, Rajdev L and Broder M (2006). Pathophysiology of bone metastases. Cancer Biol Ther. 5: 1078-1081.

Blair JM, Zheng T and Dunstan CR (2007). RANK ligand. Int. J. Biochem. Cell Biol. 39: 1077-1081.

Bonnelye E, Kung V, Laplace C, Galson DL, et al. (2002). Estrogen receptor-related receptor alpha impinges on the estrogen axis in bone: potential function in osteoporosis. Endocrinology 143: 3658-3670.

Bord S, Ireland DC, Beavan SR and Compston JE (2003). The effects of estrogen on osteoprotegerin, RANKL, and estrogen receptor expression in human osteoblasts. Bone 32: 136-141.

Caim RN, Ye KP and Jin H (2009). Research progress of osteoporosis and atherosclerosis pathogenesis. Chin. J. Osteoporosis 15: 789-792.

Gao ZY, Gao K, Shen B, Zhang H, et al. (2009). Ovariectomized rat bone receptor activator of nuclear factor factor ligand and osteoprotegerin expression changes. J. Clin. Rehabilitative Tissue Engineering Res. 13: 2877-2881.

Hamdy NA (2006). Osteoprotegerin as a potential therapy for osteoporosis. Curr. Rheumatol. Rep. 8: 50-54.

Hamdy NA (2007). Targeting the RANK/RANKL/OPG signaling pathway:a novel approach in the management of osteoporosis. Curr. Opin. Investig. Drug. 8: 299-303.

Hofbauer LC, Zeitz U, Schoppet M, Skalicky M, et al. (2009). Prevention of glucocortieoid induced bone loss in mice by inhibition of RANKL. Arthritis Rheum. 60: 1427-1437.

Kalu DN (1991). The ovariectomized rat model of postmenopausal bone loss. Bone Miner. 15:175-191.

Macmillan D, Bill RM, Sage KA, Fern D, et al. (2001). Selective in vitro glycosylation of recombinant proteins: emisynthesis of novel homogeneous glycoforms of human erythropoietin. Chem. Biol. 8: 133-145.

Marie P and Halbout P (2008). OPG/RANKL: role and therapeutic target in osteoporosis. Med Sci. 24: 105-110.

Pobeha P, Lazúrová I and Tkácová R (2010). Osteoporosis in chronic obstructive pulmonary disease. Vnitr. Lek. 56: 1142-1149.

Reid P and Holen I (2009). Pathophysiological roles of osteoprotegerin (OPG). Eur. J. Cell Biol. 88: 1-17

Rogers A, Saleh G, Hannon RA, Greenfield D, et al. (2002). Circulating estradiol and osteoprotegerin as determinants of bone turnover and bone density in postmenopausal women. J. Clin. Endocrinol. Metab. 87: 4470-4475

Saika M, Inoue D, Kido S and Matsumoto T (2001). 17 beta-estradiol stimulates expression of osteoprotegerin by a mouse stromal cellline,ST-2,via estrogen receptor-alpha. Endocrinology. 142: 2205-2212.

Sasaki N and Kusano E (2006). Bone and bone related biochemical examinations. Bone and collagen related metabolites, Measurement and clinical role of OPG. Clin. Calcium 16: 956-962.

Shimizu-Ishiura M, Kawana F and Sasaki T (2002). Osteoprotegerin administration reduces femoral bone loss in ovariectomized mice via impairment of osteoclast structure and function. J. Electron Microsc. 51: 315-325.

Sinclair AM and Elliott S (2005). Glycoengineering: the effect of glycosylation on the properties of the rapeutic proteins. J. Pharm. Sci. 94: 1626-1635.

Syed F and Khosla S (2005). Mechanisms of sex steroid effects on bone. Biochem. Biophys. Res. Commun. 328: 688-696.

Tanaka S (2007). Signaling axis in osteoclast biology and therapeutic targeting in RANKL/ RANK/OPG system. Am. J. Nephrol. 27: 466-478.

Tanaka H, Mine T, Ogasa H, Taguchi T, et al. (2011) .Expression of RANKL/OPGduring bone remodeling in vivo. Biochem Biophys Res Commun. 411: 690-694.

Thompson DD, Sirnrnons HA, Pirie CM and Ke HZ (1995). FAD Guidelines and animal models for osteoporosis. Bone 17: $125 \mathrm{~s}-133$.

Van Staa TP, Leufkens HG, Abenhaim L, Zhang B, et al. (2000). Use of oral corticosteroids and risk of fractures. J. Bone Miner. Res. 15: 993-1000. 
Wada T, Nakashima T, Hiroshi N and Penninger JM (2006). RANKL-RANK signaling in osteoclastogen-esis and bone disease. Trends Mol. Med. 12: 17-25.

Wang FS, Ko JY, Yeh DW, Ke HC, et al. (2008). Modulation of Dickkopf-1 attenuates glucocorticoid induction of osteoblastapoptosis, adipocytic differentiation and bonemass loss. Endocrinology 149: 1793-1801. 